\title{
Syphilis infection throughout the whole gastrointestinal tract
}

A 30-year-old Japanese woman presented to our hospital with epigastric pain. A physical examination revealed epigastric tenderness and red eruptions on her face. Laboratory evaluation showed normal results for leukocyte count and serum protein level, but a slightly elevated C-reactive protein (CRP) level of $1.10 \mathrm{mg} / \mathrm{dL}$. The findings on serologic tests for syphilis, including rapid plasma regain and Treponema pallidum antibody hemagglutination test, were positive.

Esophagogastroduodenoscopy (EGD) revealed hypertrophy of the rugae and multiple irregular ulcers in the stomach (৫ Fig.1). A Helicobacter pylori culture test was negative. Colonoscopy revealed reddish mucosa in the terminal ileum and slightly elevated lesions with decreased normal vascular networks in the descending colon ( $\bullet$ Fig. 2 ). Biopsy specimens were obtained from the gastric ul- cers, the red ileal mucosa, and the elevated lesions in the descending colon. Pathological analysis of these revealed regenerative epithelium and marked lymphoplasmacytic infiltration with no evidence of malignancy. Warthin-Starry staining revealed infiltration by spirochetes in the epithelium and lamina propria of the ileal and colonic tissues. No spirochetes were detected in the gastric tissues, although a polymerase chain reaction did detect the presence of some genes from $T$. pallidum with $100 \%$ similarity.

The patient was treated with penicillin (1500 mg/day) for 7 weeks, following which both her epigastric pain and the endoscopic appearances were improved. This is the first reported case of syphilis infection in which lesions were observed throughout the whole gastrointestinal tract. It has been reported that the rate of gastric involvement is only $1 \%$ [1] and syphilis infections in the colon have been detected in only a few cases [2-5]. When gastroscopic findings such as hypertrophy of the rugae and multiple irregular ulcers are observed, gastric syphilis should be considered. In addition, other sections of the gastrointestinal tract, including the small and large intestines, should be examined.

\section{Endoscopy_UCTN_Code_CCL_1AB_2AG}

\section{Competing interests: None}

\section{Masami ljiri ${ }^{1}$, Mikihiro Fujiya ${ }^{1}$, Nobuhiro Ueno', Shin Kashima', Tomohisa Watari ${ }^{2}$, Satoshi Fujii ${ }^{3}$, Toshikatu Okumura ${ }^{1}$}

${ }^{1}$ Division of Gastroenterology and Hematology/Oncology, Department of Medicine, Asahikawa Medical University, Asahikawa, Hokkaido, Japan

2 Department of Medical Laboratory and Blood Center, Asahikawa Medical University Hospital, Asahikawa, Hokkaido, Japan

3 Department of Laboratory Medicine,

Asahikawa Medical University, Asahikawa, Hokkaido, Japan
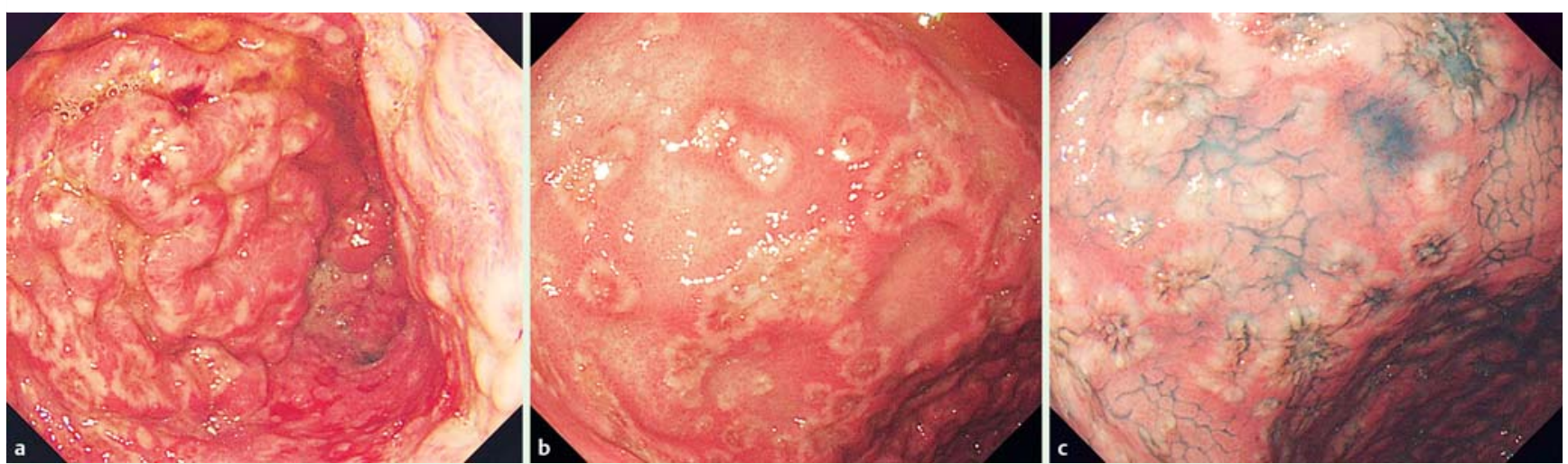

Fig. 1 Esophagogastroduodenoscopy images showing hypertrophy of the rugae and multiple irregular ulcers in the stomach.
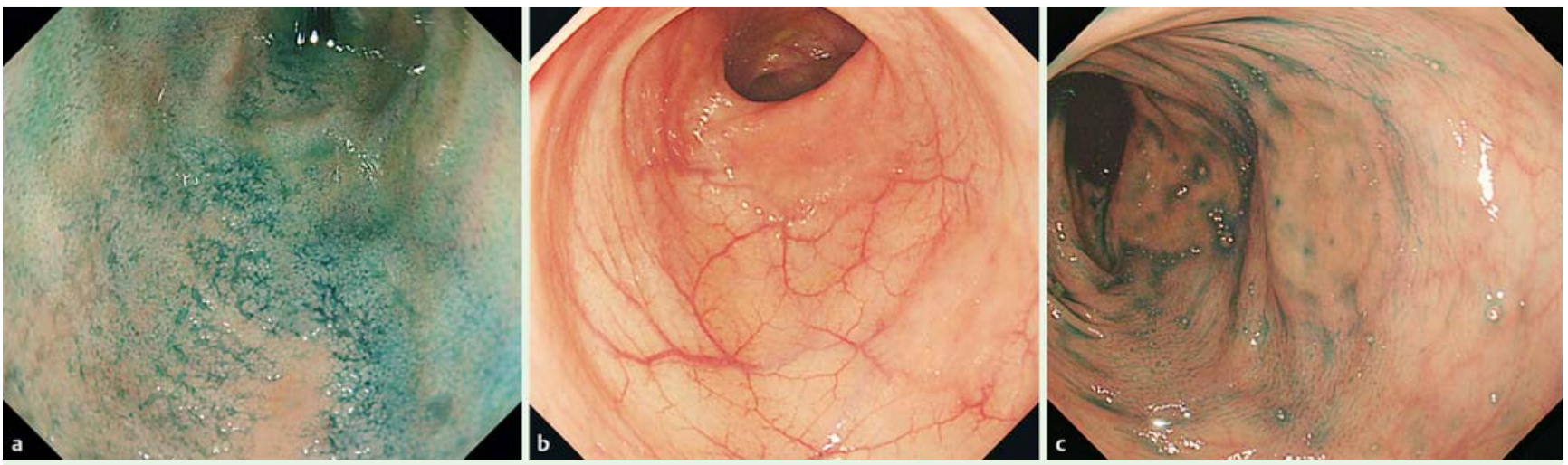

Fig. 2 Colonoscopy images showing: a reddish mucosa in the terminal ileum; $\mathbf{b}, \mathbf{c}$ slightly elevated lesions with decreased normal vascular networks in the descending colon. 


\section{References}

1 Atten MJ, Attar BM, Teopengco E et al. Gastric syphilis: a disease with multiple manifestations. Am J Gastroenterol 1994; 89: 2227 2229

2 Song SH, Jang I, Kim BS et al. A case of primary syphilis in the rectum. J Korean Med Sci 2005; 20: 886-887

3 Bassi O, Cosa G, Colavolpe A et al. Primary syphilis of the rectum - endoscopic and clinical features. Report of a case. Dis Colon Rectum 1991; 34: 1024-1026

4 Febbraro I, Manetti G, Balestrieri Pet al. Rectal cancer or rectal chancre? Beware of primary syphilis. Dig Liver Dis 2008; 40: 579-581

5 Israel GB, Leonor GR. Primary syphilitic chancre in the rectum. Endoscopy 2014; 46: E533

\section{Bibliography}

DOI http://dx.doi.org/

10.1055/s-0042-117715

Endoscopy 2016; 48: E338-E339

(c) Georg Thieme Verlag KG

Stuttgart · New York

ISSN 0013-726X
Corresponding author

Mikihiro Fujiya, MD

Division of Gastroenterology and Hematology/Oncology Department of Medicine Asahikawa Medical University 2-1-1-1, Midorigaoka-higashi Asahikawa Hokkaido 078-8510 Japan

Fax: +81-166-682469

fjym@asahikawa-med.ac.jp 